\title{
Dense Antihydrogen: Its Production and Storage to Envision Antimatter Propulsion
}

\author{
Michael Martin Nieto† Michael H. Holzscheiter $\ddagger$ and Thomas \\ J. Phillips* \\ $\dagger$ Theoretical Division MS-B285 and $\ddagger$ Physics Division MS-H803, Los Alamos \\ National Laboratory, University of California, Los Alamos, NM 87545 USA \\ * Physics Department, Duke University, Durham, NC 27708 USA \\ Email: mmn@lanl.gov, mhh@lanl.gov, phillips@physics.duke.edu
}

\begin{abstract}
We discuss the possibility that dense antihydrogen could provide a path towards a mechanism for a deep space propulsion system. We concentrate at first, as an example, on Bose-Einstein Condensate (BEC) antihydrogen. In a Bose-Einstein Condensate, matter (or antimatter) is in a coherent state analogous to photons in a laser beam, and individual atoms lose their independent identity. This allows many atoms to be stored in a small volume. In the context of recent advances in producing and controlling BECs, as well as in making antihydrogen, this could potentially provide a revolutionary path towards the efficient storage of large quantities of antimatter, perhaps eventually as a cluster or solid.
\end{abstract}

\section{Introduction}

Within this century, the challenge to develop deep space missions will be undertaken. To complete a mission within a reasonable time frame, even to the nearest objects of interest, the Oort Cloud or the Alpha Centauri star system (4.3 light years away), the velocity of the spacecraft needs to be high, up to more than $10 \%$ of the speed of light. To achieve this one needs the highest energy-density fuel conceivable. This would be antimatter; a large amount of it and in a compact form.

Antimatter can produce three orders of magnitude more energy per gram than fission or fusion and ten orders of magnitude more energy than the chemical reactions currently used for propulsion. As a result, it is a prime candidate for use in future exploration beyond the solar system. It also is a candidate for future missions to the edge of the solar system, which now require on the order of 15 years after launch to reach Pluto.

An ideal antimatter propulsion system would convert all the energy from matterantimatter annihilation into propulsion. Of course, it has long been realized that only a fraction of the total energy could be used in an antimatter propulsion system, but much thought has also gone into optimizing this fraction [1]. Further, such a system would require what is by today's standards a very large amount of stored antimatter. For a 
$500 \mathrm{~kg}$ probe, it would take more than $5 \mathrm{~g}$ of antimatter to get out to 200 Astronomical Units (past the Heliopause) in 1 year and more than $2.5 \mathrm{~kg}$ of antimatter to go 1 light year in 10 years.

Alternative propulsion schemes have also been proposed that use antimatter as a catalyst. They would require substantially less antimatter. (For example, they would use antimatter to drive fusion reactions [2] or fission reactions [3.) But for them the total weight of nuclear fuel for the same energy output is again roughly 3 orders of magnitude larger than for a pure annihilation energy source.

The amount of antimatter that can be stored per unit volume is critical to make the necessary equipment of reasonably low mass and to minimize the requirements on secondary technical support systems; i.e., cryogenic refrigerators. Unfortunately, the density of the most obvious candidate for a significant amount of stored antimatter, antiprotons, is limited by the Brillouin density [4,

$$
n_{0}=\frac{B^{2}}{8 \pi m c^{2}} \text {. }
$$

This is the limiting density for radial confinement in a magnetic field. For antiprotons stored in a 6 T magnetic field, typical for today's technology, it would be around $2 \times 10^{12}$ $\mathrm{cm}^{-3}$. Therefore, to achieve high storage densities, it will be necessary to make and store neutral antimatter. The only realistic neutral candidate is antihydrogen.

Further, a Bose-Einstein Condensate (BEC) of antihydrogen, even with present day BEC hydrogen densities, would be orders of magnitude more dense than the Brillouin storage density limit for charged antiprotons. This latter is itself orders of magnitude higher than the highest antiproton density so far achieved, $\sim 10^{6} \mathrm{~cm}^{-3}[\underline{5}, 6]$. To make a BEC of antihydrogen would be an individual step, where one could learn the techniques of controlling a relatively large amount of antihydrogen. The clear ultimate goal would be to make very dense antihydrogen, in the form of clusters or solids (perhaps stored diamagnetically).

Therefore, since a) controlled antiprotons have been created, b) cold antihydrogen has just been made, c) controlled antihydrogen is envisioned in the foreseeable future, and d) high-density BEC hydrogen has also been made (see the next paragraph), we find it appropriate to consider the possibility of producing antihydrogen BEC as a first, but critical, step towards the potential use of antimatter in a propulsion system.

Fundamental quantum theory predicts that there are ordinarily two kinds of particles; i) Fermions, which are particles with half-integer internal quantum angular momenta (like electrons and protons) and ii) Bosons, which are particles with integer internal quantum angular momenta (like photons or even numbers of Fermions such as the hydrogen atom). From the early work of Bose and Einstein, it was realized that many Bosons, if cool enough, could all fall into the lowest allowed quantum energy and behave as one single (even macroscopic) quantum object with remarkable properties. Two well-known examples are superconductivity, a sea of paired electrons, and superfluids, usually a liquid of ${ }^{4} \mathrm{He}$ atoms (two protons, two neutrons, and two electrons). The recent discovery that atomic hydrogen can be condensed into a Bose-Einstein state, opened up 
new possibilities.

The purpose of this communication is to present an initial evaluation of possible routes towards efficient production and long-term storage of antimatter and also to identify the key issues that need to be addressed, specifically using BEC antihydrogen. We note that much of the equipment and the techniques that would be needed to study some of these concepts are already commonly available. Further, because of the symmetry between matter and antimatter, one should be able to perform many of the studies with matter. This is important since antiprotons are not (yet) readily available in large quantities.

\section{Antihydrogen}

Antihydrogen is the antimatter equivalent of hydrogen. It is composed of a positron in an atomic orbit around an antiproton. Antihydrogen is produced if a free antiproton $(\bar{p})$ picks up a positron $\left(e^{+}\right)$in an atomic orbit.

The antiprotons for antihydrogen can be made in high-energy collisions. They are currently being produced at the Fermilab and CERN particle accelerators for use in high-energy physics experiments. Antiprotons at CERN have been slowed and captured in Penning traps [7, 8]. The collection process is currently quite inefficient, but this efficiency could be improved by several orders of magnitude with straightforward changes 9]. The positrons for antihydrogen are much more easily made using either small electron accelerators [10] or collected from certain radioactive sources with well-known gas moderation techniques [1].

Previously, a few atoms of antihydrogen have been produced at CERN [12] and Fermilab [13, but these have been produced at relativistic speeds, much too fast to capture. However, the ATHENA experiment at CERN recently produced a significant amount (of order 50,000) of cold antihydrogen atoms [14, a feat which caused international excitement [15]. Later, the ATRAP collaboration also reported production of antihydrogen [16].

The antihydrogen program at CERN [17, 18] is geared towards tests of fundamental physics principles such as spectroscopic studies of CPT invariance and the weak equivalence principle. While the aim of that research is to produce, what is for our purposes, a moderate number of anti-atoms, much can be learned from it about the different production and storage mechanisms proposed for our present purposes.

\section{Production of Cold Antihydrogen}

For any scheme proposed to produce slow antihydrogen, the velocity of the positron and antiproton relative to each other must be very small. This requirement is best described in terms of a temperature of the order of several Kelvin, describing the energy distribution in the center-of-mass frame. Such temperatures can be achieved by trapping the positrons and antiprotons in Penning traps. These traps confine charged particles 
in the radial direction with a strong solenoidal magnetic field (typically of the order of several Tesla), and in the longitudinal direction with static electric fields.

Once either positrons or electrons have been captured in a Penning trap, they cool very rapidly to equilibrium with the ambient temperature of the trap by emitting synchrotron radiation. In a 6 Tesla magnetic field the time constant for this process is approximately one hundred milliseconds.

Antiprotons have a much slower time constant for cooling through synchrotron radiation due to the heavier mass, but they can be cooled by loading electrons in the same trap. Coulomb collisions between the electrons and the antiprotons lead to thermalization of the two species, thus cooling the antiprotons. The heat input to the cold electrons due to this process is continuously removed by synchrotron radiation into the heat sink of the cryogenic environment of the experimental apparatus. Therefore, the velocities of all particles involved in this process can be reduced by cooling the physical structure of the trap to cryogenic temperatures.

This technique was first introduced by Rolston et al. [19] and has been successfully used by two experiments at CERN, PS196 [20] and PS200 [6]. The latter experiment currently holds the world record for the number of cold antiprotons confined in a trap of 1 million [5]. The PS200 technology has now been fully incorporated into the ATHENA experiment at CERN [14, 17].

\section{Processes to Combine Antiprotons and Positrons into Antihydrogen}

When an antiproton and a positron come together, they can only become an antihydrogen atom if there is a mechanism to remove energy from the system. This binding energy can be removed either by the radiation of a photon (radiative recombination) or it can be carried off by a third particle (three-body recombination). A number of different channels have been considered for such processes [21].

One of the strongest candidates for our purpose appears to be the three-body recombination (TBR) reaction $\bar{p}+2 e^{+} \rightarrow \bar{H}+e^{+}$. The calculated rate for this reaction can be quite high [22]:

$$
\Gamma=6 \times 10^{-13}\left(\frac{4.2}{T}\right)^{9 / 2} n_{e}^{2}\left[\mathrm{~s}^{-1}\right]
$$

per antiproton, where $n_{e}$ is the positron density in number per $\mathrm{cm}^{3}$ and $T$ is the temperature. For a positron density of $10^{8} \mathrm{~cm}^{-3}$ at $4.2^{\circ} \mathrm{K}$ the half life for an antiproton in the positron plasma before it is converted into a antihydrogen atom is $170 \mu \mathrm{s}$. This essentially promises an instantaneous conversion of all antiprotons into antihydrogen atoms.

But most of these atoms are formed in highly excited Rydberg states, and it currently is not clear what will be the effect of the electric and magnetic fields in the trap environment on the net production rate. The recombination rate observed to date by the ATRAP collaboration is significantly lower than the predictions from equation (2). Most likely this is due to field ionization of the higher lying Rydberg states. To overcome 
this problem and to increase the net production rate it will be necessary to de-excite these atoms with an appropriate laser. Care must then be taken that photo-ionization of the Rydberg atoms does not limit the net production.

A second production mechanism of interest to us is the spontaneous radiative recombination (SRR) in which a positron is bound to an antiproton through emission of a photon. In this process $60 \%$ of the antihydrogen atoms are formed in states $n=1$ through 10 and $20 \%$ of the atoms are formed directly in the ground state. The recombination coefficient $\alpha^{S R R}=\left\langle\sigma^{S R R}(v) v\right\rangle$ for this process (at a positron temperature of $0.1 \mathrm{meV}$ ) is of the order of $10^{-10} \mathrm{~cm}^{3} / \mathrm{s}$. This recombination process is therefore substantially slower than the theoretical three-body reaction at the same low temperature. But it produces atoms stable against reionization by the strong electromagnetic fields in the environment. Additionally, the recombination rate can be enhanced by using a laser to stimulate a transition into a specific, stable, low-lying $n$ level.

Assuming the parameters proposed for the ATHENA experiment of $10^{7}$ antiprotons and $10^{8}$ positrons confined to a volume of $1 \mathrm{~cm}^{3}$, this reaction yields a production rate of 90,000 antihydrogen atoms per second. The entire sample of $10^{7}$ antiprotons would be converted to antihydrogen in less than 20 minutes. Considering the fact that the long range goal will be to store the produced antihydrogen for extended periods of time this rate is fully sufficient for initial experiments.

Several ideas to enhance the net production rate for both of the above mechanisms have been discussed in the literature [21].

\section{Experimental Methods for Antihydrogen Production}

Positrons and antiprotons have opposite charge, so they must be trapped in potential wells of opposite signs in Penning traps. Several ideas have been discussed in the literature on how to make the two oppositely charged clouds physically overlap in space, either in static configurations or by moving the electric wells through one another. The simplest rendition of such a scheme is the "nested trap" 23. In its most straight forward realization it consists of a sequence of five cylindrical electrodes that are biased in such a way that an elongated potential well is formed for antiprotons. This well which has a hill in its center that can then serve as a well for the oppositely charged positrons. (See Figure 1.)

To overcome the possible separation of the oppositely charged plasmas, the ATHENA collaboration uses an approach where the antiproton cloud is released from a side well into a positron plasma. The density and length of the positron plasma is chosen so that an individual low-energy antiproton entering the positron plasma along the axis will come to rest before leaving the plasma column again. Therefore, it will recombine with high probability in a single passage.

All of the above considerations have been successfully incorporated in the ATHENA experiment, which recently detected the creation of around 50,000 cold anti-hydrogen 


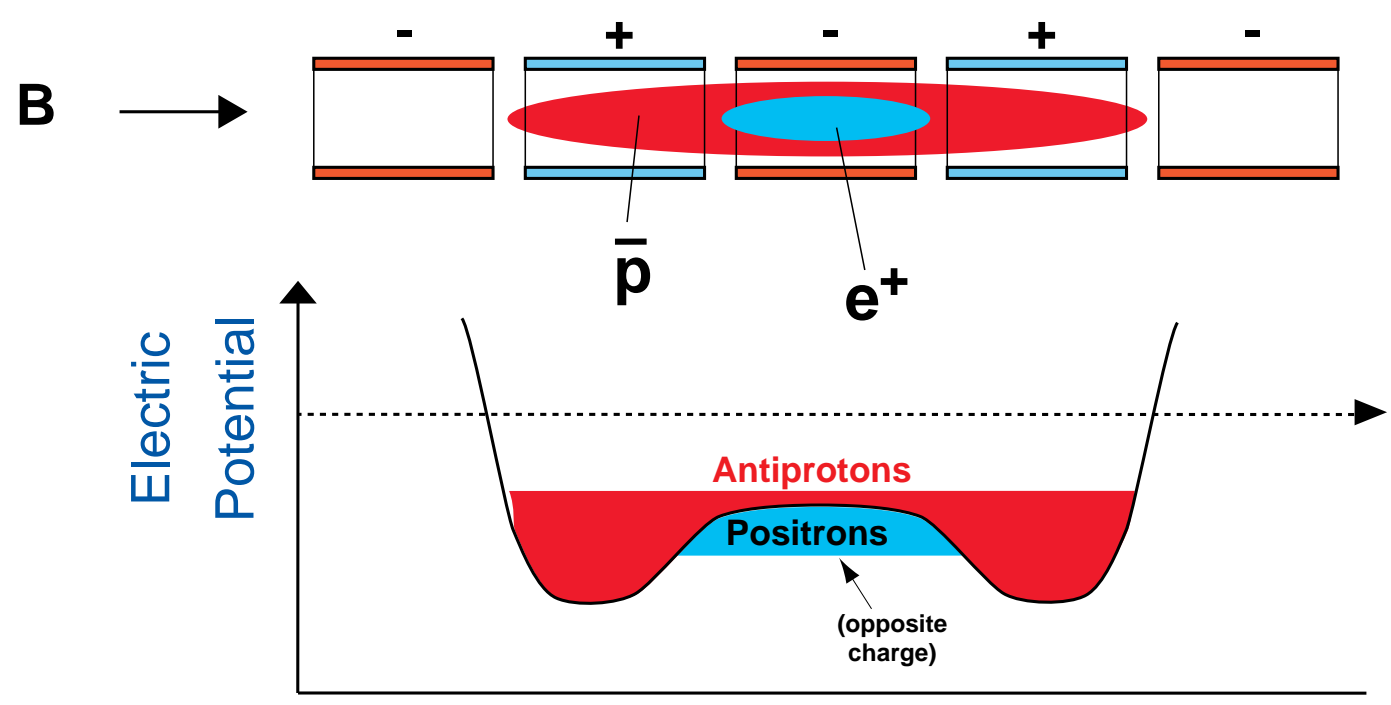

Figure 1. Physical realization and the electric potential of a nested trap to store antiprotons and positrons in the same location.

atoms at CERN [14]. The creation was demonstrated by the detection of the annihilation products, preferentially on the walls of the trap. (See Figure 2, This completes a major step on the road to the envisioned technology.

Once antihydrogen has been formed it will no longer be trapped in the Penning trap configuration. It will escape in the direction of the antiproton's initial velocity and most likely will annihilate at the wall of the trap. This will make it difficult to diagnose the dynamics of the recombination reaction and the dependence of the recombination rate on the many different parameters relevant in these experiments. While recombination at rest will be an important part of an experiment aimed at filling some form of neutral storage scheme, we feel a method allowing easier diagnostics schemes is appropriate for the first stages of development.

Observe that it is the relative velocity between positrons and antiprotons which drives the reaction rate. The velocity of a positron at 4 Kelvin is about $1.1 \times 10^{4} \mathrm{~m} / \mathrm{sec}$. An antiproton of the same velocity has an equivalent energy of about $0.6 \mathrm{eV}$ (or 7300 $\mathrm{K})$. This allows one to accelerate antiprotons into or through positron clouds without diminishing the conversion rate to antihydrogen.

Because of this, an appropriate proposal to make antihydrogen starts by keeping the antiprotons and positrons in separate potential wells in a Penning trap, as shown in Figure 3. The potential of the antiprotons can be raised with a small applied voltage so that when the antiprotons are released, they are accelerated along the axis of the trap. When they enter the positron plasma, some antiprotons will pick up positrons to form antihydrogen. The neutral antihydrogen will no longer be confined, so it will exit the trap along the axis, making a beam of antihydrogen.

For sufficiently large positron densities, the production efficiency should be very 


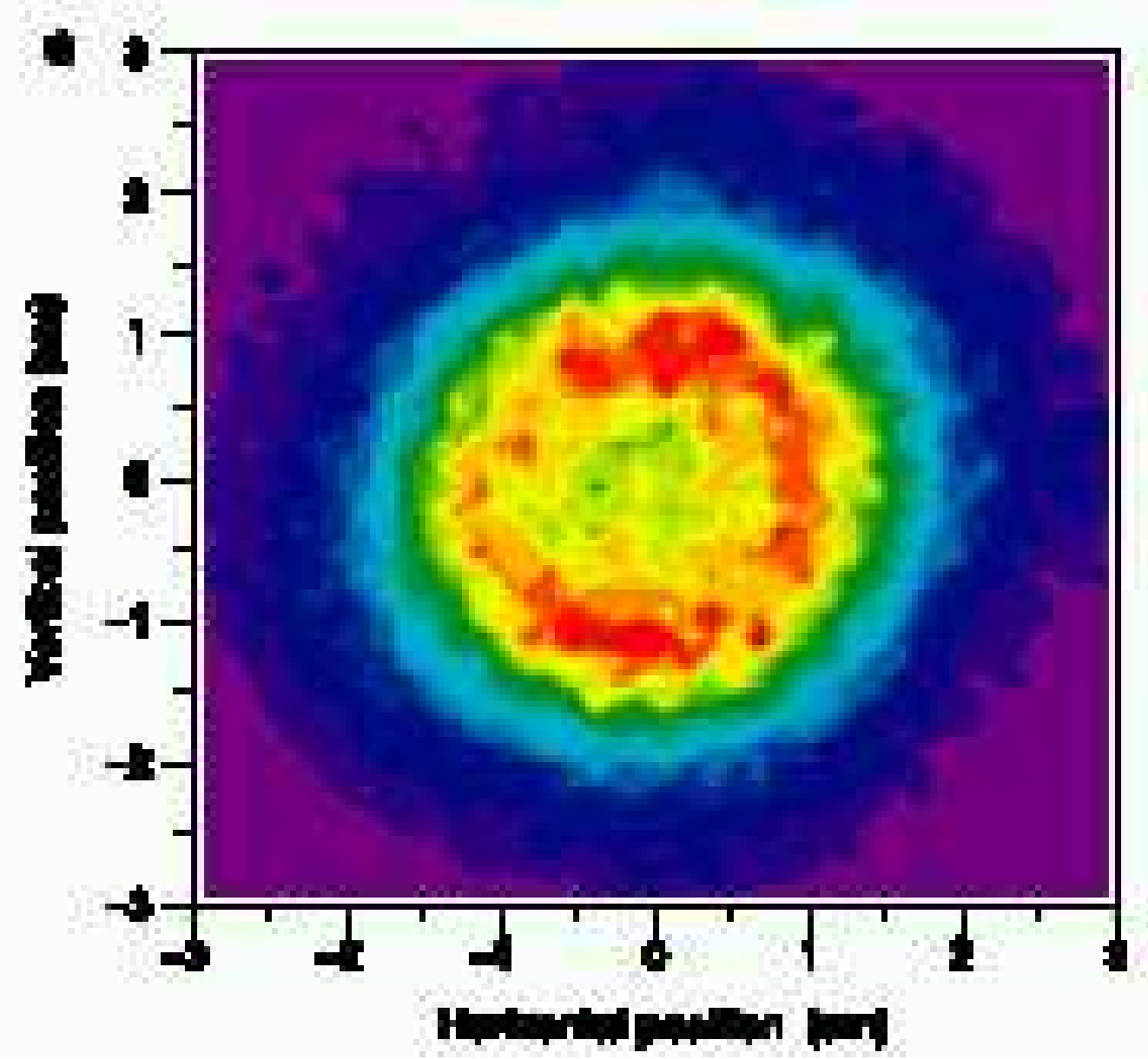

Figure 2. Contour plot of the distribution (obtained by projecting onto the plane perpendicular to the magnetic field) of the vertex positions of reconstructed anti-hydrogen annihilation events from the ATHENA cold antihydrogen production experiment [14].

high. To produce a well defined beam, the initial velocity of the antiprotons and the density and length of the positron plasma must be carefully chosen.

\section{Antihydrogen Storage}

Long term storage of substantial amounts of antimatter must be developed to enable space missions relying on antimatter-based propulsion systems. Although it is clear that ultimately neutral antimatter must be used, up to now, no valid long-term storage concept for large quantities of antihydrogen has been developed. On the other hand, BEC confinement of neutral spin-polarized hydrogen atoms at densities up to $5 \times 10^{15}$ $\mathrm{cm}^{-3}$ has been demonstrated. [24. We believe that developing the tools for making antihydrogen BEC could be an important step towards solving the antimatter storage problem.

While storage of high density samples of neutral alkali atoms has been demonstrated 


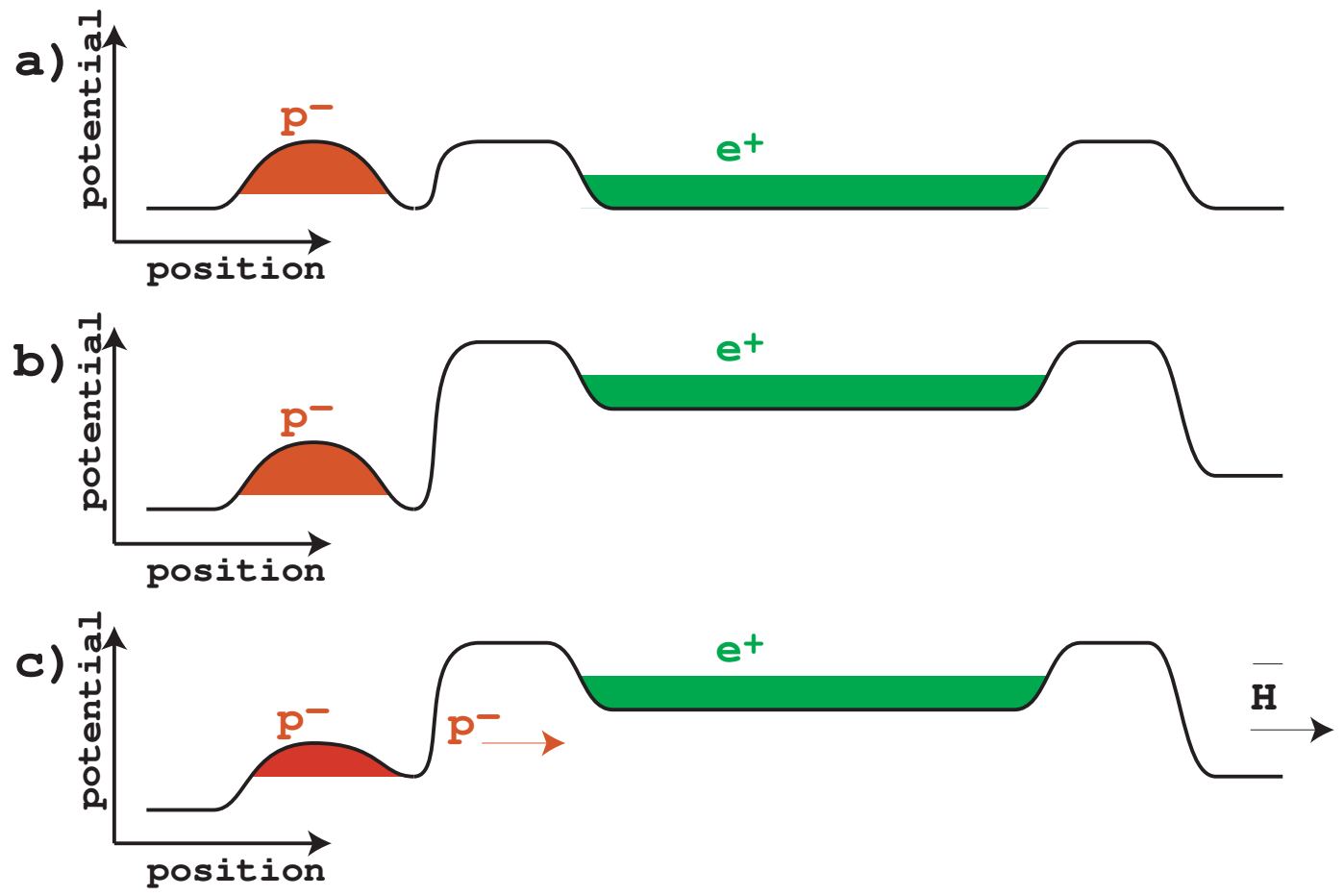

Figure 3. To make a beam of antihydrogen, (a) cold antiprotons and positrons are first stored in separate potential wells in a penning trap. The figure plots electrical potential on the vertical axis but because of their negative charge, the potential energy for antiprotons is inverted relative to the electric potential and they can be trapped under peaks in the electric potential. One can also look at the figure upside down. In (b), a small voltage is applied between the antiprotons and the positrons. So, when in (c) the right side of the potential barrier confining the antiprotons is lowered (raised with respect to the positrons), the antiprotons are accelerated. They pass through the positron plasma with their momentum directed down the axis of the trap. If the density of positrons is high enough, some antiprotons will pick up positrons and exit the trap as a beam of antihydrogen.

using magneto-optical and optical traps, the lack of appropriate laser systems has so far precluded hydrogen from such methods. Nevertheless, the BECs with the largest total number of particles have been formed using hydrogen. Until now neutral hydrogen has been stored either in cryogenic cells, by using wall collisions as the confinement mechanism, or in magnetic traps employing static magnetic gradient fields [25, 26]. Although total quantum reflection at containment walls is a possibility, without such a new technology, wall collisions are clearly unacceptable for the case of antihydrogen. One then needs to consider magnetic traps.

A static magnetic field trap is constructed with a minimum at its center. Then the only states that can be trapped are the so-called low-field seeking states. (The upper two states in the Hyperfine diagram for the ground state of atomic hydrogen.) These states will decay by the mechanism of dipole relaxation to the lower, un-trapped states with a rate constant of $\sim 10^{-15} \mathrm{~cm}^{-3} \mathrm{~s}^{-1}$ [27, 28]. Another (smaller) loss mechanism is three-body recombination, which for hydrogen is $\left(\sim 10^{-19} \mathrm{~cm}^{-3} \mathrm{~s}^{-1}\right)$ [29]. Apparently, 
these effects would strongly limit the density of achievable BECs.

But there are a number of other considerations that mean a controlled dense BEC is not ruled out in principle. Firstly, it is interesting to note that in a BEC, the coherence properties reduce the above dipole and three-body transition rates by factors of 2 and 6 , respectively [30]. Such a factor has been verified experimentally using Rubidium [31.

Next, Lovelace et al. showed [32, 33] that a trap confining all hyperfine states of neutral (anti)hydrogen can be constructed using AC magnetic fields. (The situation is similar to strong focusing in particle accelerators.) Such a trap has not yet been experimentally realized because i) magnetostatic traps have been entirely sufficient for current research interests and ii) the technical challenges presented in generating AC magnetic fields with the appropriate amplitude and frequency are sizable. However, especially given the proper interest, traps of this type are certainly feasible.

But most importantly, the above transition rates are for current types of traps, with magnetic fields on the order of up to a few $\mathrm{T}$. The rates are strongly magnetic-field dependent, and disappear for very large magnetic fields, of order $140 \mathrm{~T}$ [29, 34]. Fields on this order can now be made in a pulsed mode. Efforts to eventually create them statically have commercially reached $21 \mathrm{~T}$ [35].

At present the wasteful method of resonant evaporative cooling is used to achieve the temperatures and densities needed to form a hydrogen BEC. But the development of lasers for direct and efficient cooling of hydrogen atoms has now just started. Efficient laser cooling of hydrogen will revolutionize the methodology of forming, controlling, and studying hydrogen Bose-Einstein Condensates. All the cooling, as well as the optical storage, methods that have been so successful with Alkali BECs could then be used with antihydrogen. That is to say, once an antihydrogen atom has been made and stored, in principle the only additional requirement (beyond those needed for normal hydrogen) is the requirement that the residual pressure and temperature be low enough so that no residual normal atom be around to annihilate an antihydrogen.

Further, enormous advances have been achieved in the control of Alkali BECs leading to the demonstration of the coherent control of a BEC and the demonstration of the concept of atom-lasers [36]. Based on these developments a concept of coherently amplified antimatter waves could be envisioned to increase antihydrogen storage capabilities by a significant factor. Such schemes are far beyond current technological capabilities, but the study of the underlying physical principles is a necessary beginning if one is to hope for a breakthrough discovery.

If the envisioned progress comes to fruition, laser cooling could then be used in an attempt to efficiently make an antihydrogen BEC.

\section{Conclusion}

An antihydrogen BEC would be an important step down a path that could eventually lead to even more dense anti-hydrogen molecules, liquids, solids, and cluster ions [37]39. Indeed, since one might expect the next stage to be going from controlled ultra-cold 
(below $50 \mu \mathrm{K}$ ) BEC hydrogen atoms to controlled hydrogen molecules, it is heartening that there is evidence of a hydrogen-molecule superfluid with a critical temperature of $0.15 \mathrm{~K}$ [40]. Since the triple point of hydrogen is at $13.8 \mathrm{~K}$, a potential path to denser condensed antimatter becomes more interesting.

In our opinion a space-certified storage system for neutral antimatter can not be obtained from a linear extrapolation of heretofore existing technologies. Rather, it requires a scientific and/or technological breakthrough of the kind that Bose-Einstein Condensation might provide. While breakthroughs can never be predicted, they typically will not happen without the definition of a strong need and the challenge presented to the scientific community by a truly ambitious goal. Meanwhile many of the underlying issues can be addressed with both the modest supply of antimatter available at this time at accelerator centers world wide and with the limited means to store the particles. The technological and scientific knowledge gained in these tests will enable us to lay out a path into the future of antimatter-based propulsion systems.

Current antiproton production rates are low. While clever techniques can enhance these rates by several order of magnitude and quantities sufficient for advanced concepts can be produced given enough economic and political pressure onto the few available sources, a real breakthrough can only come through continued interest and research in this area. A good analogy is the comparison between a light bulb and a laser. In both cases light is produced, but in one system through thermal heating of a material and in the other through coherent processes. Antiprotons are currently produced by heating a metal target with a primary proton beam. This is a direct analogy to the light bulb we are still awaiting the invention of a 'laser-equivalent' for the production of particles of antimatter.

Similarly, the efficient production and storage of antihydrogen (perhaps as a BEC, cluster, or solid), which are necessary steps towards large large-scale production as a fuel, can be studied with the quantities of antihydrogen soon to be available to us. The new modest production at CERN [14] should help us in identifying key problems and in developing technologies in preparation for future higher production rates of antimatter.

CERN is the only choice for the location to make the initial scientific advances. There are a number of steps needed to create and understand the desired form of antihydrogen. (i) Trap and cool the antihydrogen in a magnetic trap, developing tunable Lyman-alpha lasers along the way. After this first step one could (iiA) convert the antihydrogen into a BEC. Alternatively, one could (iiB) form and control molecular antihydrogen, and finally (iii) convert the antihydrogen into a superconducting cluster and/or solid of antihydrogen. Each of these advances, with strong funding, might be made in on the order of 5-10 years.

Future, even moderate, sources of antihydrogen (say at GSI or Fermilab) would also enable research on antimatter-based propulsion. One could also consider the use of antimatter to drive fusion reactions [2] or fission reactions [3] and also study crucial issues necessary to develop a propulsion system based entirely upon antimatter annihilation.

To meet the challenge of interstellar travel we have to overcome enormous 
scientific and technological barriers. Antimatter-matter annihilation is one of the prime candidates to achieve the high specific impulse i) desired for the challenging missions of exploring the Heliopause and visiting the Oort Cloud, and ii) needed if we plan to attempt a rendezvous with the nearest star systems. While no clear pathway to the necessary technologies exists, possible concepts can be identified based upon the need for the highest possible energy densities.

The experimental development of such systems as BECs and atom lasers in the normal matter world of laboratory-sized research equipment can help us to reach these most ambitious goals. To achieve them quickly it is necessary to set ourselves in motion now.

\section{Acknowledgements}

We thank Michael Charlton, Lee Collins, Terry Goldman, TJ Johnson, Peter Milonni, Slava Turyshev, and especially Eddy Timmermans for their helpful comments and information. We all (MMN, MHH, and TJP) acknowledge the support of the US Department of Energy.

\section{References}

[1] Augenstein B W, Bonner B E, Mills R E, and Nieto, M M 1988 Antimatter Science and Technology (Singapore: World Scientific). See, especially, Group. III Papers, "Applied Science and Technology."

[2] Kammash T 1998 NIAC 98-02 Final Report, available at http://peaches.niac.usra.edu/studies/study_master.jsp?action=Call

[3] Lewis R A, Smith G A, Cardiff E, Dundore B, Fulmer J, Watson B J, and Chakrabarti S 1997 Space Technology and Applications International Forum. (STAIF-97), ed. El-Genk M S, AIP Conference Proceedings No. 387, Pt. 3, (New York: American Institute of Physics), pp. 14991504

[4] Brillouin L 1967 Phys. Rev. 67 260-266

[5] Holzscheiter M H, Feng X, Goldman T, King N S P, Lewis R A, Nieto M M, and Smith G A 1996 Phys. Lett. A $\mathbf{2 1 4} 279-284$

[6] Nieto M M and Holzscheiter M H 1995 Appl. Phys. B 60 103-112

[7] Holzscheiter M H et al. 1993 Nucl. Phys. A 558 709c-718c

[8] Gabrielse G et al. 1989 Phys. Rev. Lett. 63 1360-1363

[9] Jackson G 2001 in: Proceedings of the Pbar2000 Workshop, eds. Kaplan D and Rubin H (Chicago: Illinois Institute of Technology), pp. 75-84

[10] Costello D G, Groce D E, Herring D F, and McGowan J W 1972 Phys. Rev. B 5 1433-1436

[11] Surko C M, Leventhal M, and Passner A 1989 Phys. Rev. Lett. 62 901-904

[12] Baur G et al. 1996 Phys. Lett. B 368 251-258

[13] Blanford G et al. 1998 Phys. Rev. Lett. 80 3037-3040

[14] Amoretti M, Amsler C, Bonomi G, Bouchta A, Bowe P, Carraro C, Cesar C L, Charlton M, Collier M J T, Doser M, Filippini V, Fine K S, Fontana A, Fujiwara M C, Funakoshi R, Genova P, Hangst J S, Hayano R S, Holzscheiter M H, Jørgensen L V, Lagomarsino V, Landua R, Lindelof D, Rizzini E L, Macri M, Madsen N, Manuzio G, Marchesotti M, Montagna P, Pruys H, Regenfus C, Riedler P, Rochet J, Rotondi A, Rouleau G, Testera G, Variola A, Watson T L, and van der Werf D P 2002 Nature 419 456-459 
[15] http://athena-positrons.web.cern.ch/ATHENA-positrons/wwwathena/inthenews.html)

[16] Gabrielse G, Bowden N S, Oxley P, Speck A, Storry C H, Tan J N, Wessels M, Grzonka D, Oelert W, Schepers G, Sefzick T, Walz J, Pittner H, Hansch T W, and Hessels E A 2002 Phys Rev. Lett. 89 233401-233404

[17] Amsler C et al. 2001 in: The Hydrogen Atom: Precision Physics of Simple Atomic Systems, eds. Karshenboim S, Pavone R, Bassani G, Inguscio M, and Hänsch T (Berlin: Springer) pp. 569-588

[18] Gabrielse G et al. 1997 CERN report CERN-SPSC-07-8; SPSC P 306 (Geneva, Switzerland)

[19] Rolston S L and Gabrielse G 1988 Hyperfine Int. 44 233-245

[20] Kalinowsky H 1992 Sov. J. Nucl. Phys. 55 848-855 [Yadernaya Fizika 55 1541-1554

[21] Holzscheiter M H and Charlton M 1999 Rep. Prog. Phys. 62 1-60

[22] Glinsky M E and O'Neil T M 1991 Phys. Fluids B 3 1279-1293

[23] Hall D S and Gabrielse G 1996 Phys. Rev. Lett. 77 1962-1965

[24] Fried D G, Killian T C, Willmann L, Landhuis D, Moss S C, Kleppner D, and Greytak T 1998 Phys. Rev. Lett. 81 3811-3814

[25] Migdall A J, Prodan J V, Phillips W D, Bergerman T H, and Metcalf H J 1985 Phys. Rev. Lett. 54 2596-2599

[26] Hess H F, Kochanski G P, Masuhara N, Kleppner D, and Greytak T J 1987 Phys. Rev. Lett. 59 $672-675$

[27] Stoof H T C, Koelman J M V A, and Verhaar B J 1988 Phys. Rev. B 38 4688-4697

[28] Masuhara N, Doyle J M, Sandberg J C, Kleppner D, Greytak T J, Hess H F, and Kochanski G P 1988 Phys. Rev. Lett. 61 935-938

[29] Gillaspy J D, Silvera I F, and Brooks J S 1989 Phys. Rev. B 40 210-223

[30] Kagan Yu, Svistunov B V, and Shlyapnikov GV 1985 JETP Lett. 42 209-212 [Pis'ma Zh. Eksp. Teore. Fiz. 42 169-172]

[31] Burt E A, Ghrist R W, Myatt C J, Holland M J, Cornell E A, and Wieman C E 1997 Phys. Rev. Lett. 79 337-340

[32] Lovelace R V E, Mehanian C, Tommila T J, and Lee D M 1985 Nature 318 30-36

[33] Lovelace R V E and Tommila T J 1987 Phys. Rev. A 35 3597-3606

[34] Stoof H T C, de Goey L P H, Verhaar B J, and Glöckle W 1988 Phys. Rev. B 38 11221-11224

[35] Oxford Instruments offers standard, vertical bore, high-resolution NMR magnet systems up to 21.1 Tesla, operating at $2.2 \mathrm{~K}$. See http://www.oxint.com

[36] Inouye S, Low R F, Gupta S, Pfau T, Gorlitz A, Gustavson T L, Pritchard D E, and Ketterle H 2000 Phys. Rev. Lett. 85 4225-4228

[37] Silvera I F 1980 the condensed phase: Fundamentals and static properties," Rev. Mod. Phys. 52 393-452

[38] Stwalley W C 1988 in: [1, pp. 373-392

[39] Young D A 1991 Phase Diagrams of the Elements, (Berkeley: University of California Press) Chap. 4, "Hydrogen."

[40] Grebenev S, Sartakov B, Toennies J P, and Villesov A F 2000 Science 289 1532-1535 